$$
\begin{gathered}
\text { Submitred to: Cleo } 2002 . \\
13 \text { Nor. 2001. }
\end{gathered}
$$

\title{
Continuous wave ultra violet laser induced frustration of etching in congruent lithium niobate
}

\author{
S. Mailis, C. Riziotis, R. W. Eason
}

Surface relief patterns have been fabricated on the $-\mathrm{z}$ face of congruent lithium niobate single crystals by illumination of the surface with continuous wave $244 \mathrm{~nm}$ laser radiation followed by chemical etching-with hydrofluoric acid.

Light induced frustration of etching (LIFE) describes the effect of the partial suppression or complete cancellation of chemical etching in the presence of light. This effect has been demonstrated in iron doped congruent lithium niobate single crystals where the $-\mathrm{z}$ face of a $\mathrm{z}$-cut crystal which etches normally (in 1:2 $\mathrm{HF}: \mathrm{HNO}_{3}$ acid mixture) can be made etch resistant in the presence of a blue laser beam during the chemical etching procedure [1]. The effect has been attributed to the generation of charges due to photoconduction which diffuse to the surface and stop the chemical etching process. Some degree of latency has also been reported [2].

Latent etch frustration effects have also been observed on the $-z$ face of undoped congruent lithium niobate after exposing the crystal surface to pulsed UV laser radiation [3].

Here we report on the latent frustration of etching which has been observed on the $-z$ face of undoped congruent lithium niobate single crystals induced by prior illumination with a frequency doubled $\mathrm{Ar}+$ continuous wave laser at $244 \mathrm{~nm}$. It is a latent effect as the laser beam does not have to be present during the etching procedure in $\mathrm{HF}$ acid and enables the fabrication of surface relief micron-size features in a simple exposure-development manner.

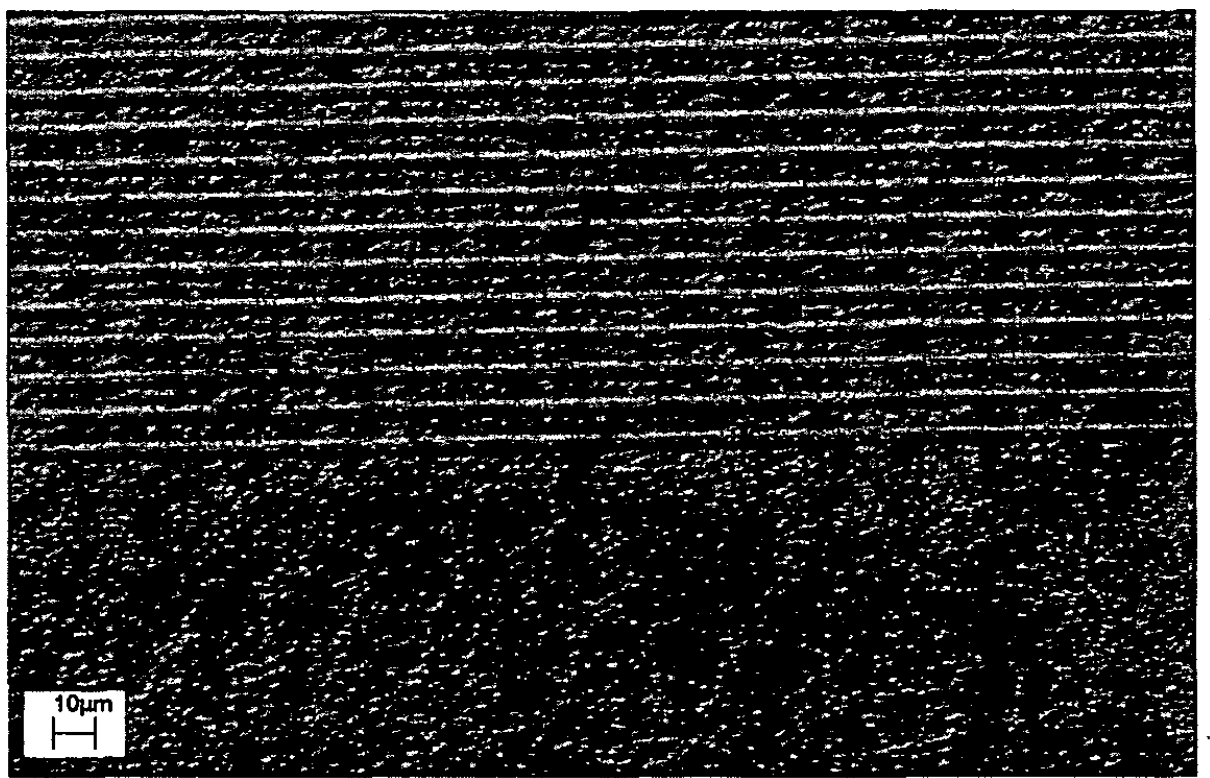

Figure 1 Scanning electron microscopy picture of a periodic relief structure on the $-z$ face of lithium niobate crystal fabricated by UV exposure followed by HF etching. The period of the pattem is $10 \mu \mathrm{m}$ and the width of each line is $3 \mu \mathrm{m}$.

Such structures are shown in the scanning electron microscope (SEM) photograph of a UV exposed/etched lithium niobate sample presented in figure 1. The top half of the SEM photograph shows a set of $3 \mu \mathrm{m}$ wide horizontal ridges which are periodically arranged at a $10 \mu \mathrm{m}$ period while the bottom half is flat showing just the surface roughness due to chemical etching. The UV exposure was performed by scanning the $-\mathrm{z}$ face of the lithium niobate sample under a focused frequency doubled $\mathrm{Ar}+$ continuous wave laser beam using high precision computer 
controlled translation stages (Aerotech ABL 800020) with positional accuracy (defined by optical encoder) of $40 \mathrm{~nm}$ at a scanning speed of $3000 \mathrm{num} / \mathrm{min}$. The laser power used was $50 \mathrm{~mW}$ and the beam spot size on the surface of the sample was $5 \mu \mathrm{m}$. The sample was then etched in $48 \% \mathrm{HF}$ acid for $1 \mathrm{hr}$ at room temperature. The periodic ridges that were revealed after the chemical etching corresponds exactly to the illuminating beam track.

The height of these surface relief patterns was about $2 \mu \mathrm{m}$ having an aspect ratio of $2 / 3$ after $1 \mathrm{hr}$ of etching as shown in the alpha step scan presented in figure 2 . The alpha step scan was taken at an oblique angle with respect to the periodic ridges to compensate for the poor spatial resolution of the instrument. This height difference after etching between the illuminated and un-illuminated areas suggests total frustration of etching (as the $-z$ face etches normally in $48 \% \mathrm{HF}$ acid at a rate of $\sim 1.7 \mu \mathrm{m} / \mathrm{hr}$ at room temperature).

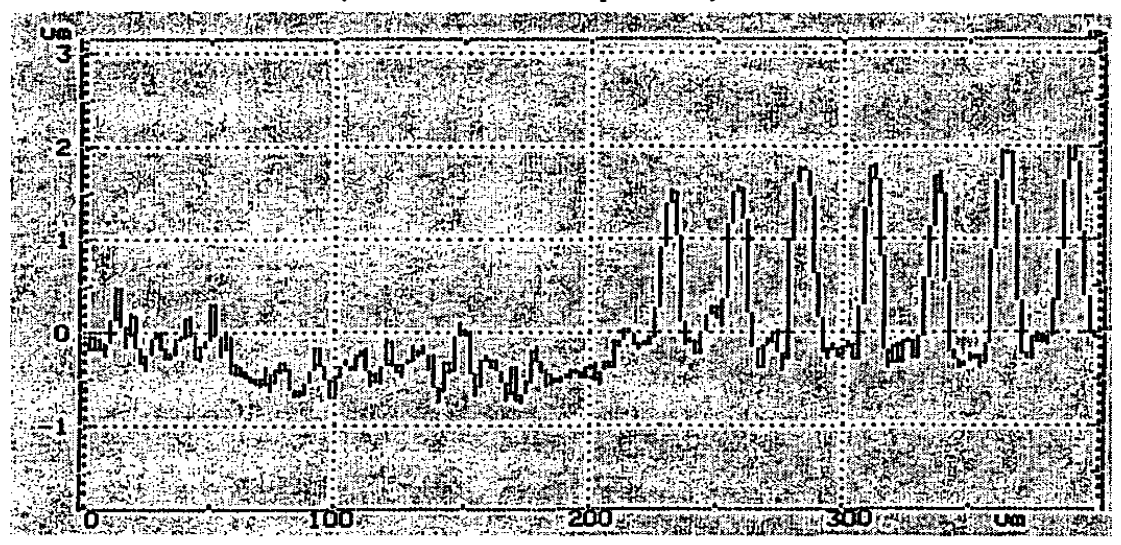

Figure 2 Alpha-step scan of the same sample taken from bottom to top (as shown in the SEM picture) at an oblique angle with respect to the ridges.

Preliminary examination of the etch frustrated area using micro-Raman analysis showed small broadening and shifting of some spectral lines, when compared with the unaffected material, suggesting structural modifications of the surface. Further investigation of the nature of the etch frustration as well as potential application in Quasi Phase Matched nonlinear devices (QPM) is currently under way.

1. I.E Barry, R.W. Eason, G. Cook "Light-induced frustration of etching in Fe-doped LiNbO," Appl. Surf. Sci. 143, 328-331 (1999)

2. A.J. Boyland, S. Mailis, I.E. Barry, RW Eason, M. Kaczmarek "I atency effects and periodic structures in light-induced frustrated etching of Fe : doped LiNbO," Appl. Phys. Lett. 77,: 2792-2794 (2000)

3. S. Mailis, P. T. Brown, C. L. Sones, I. Zergioti, R. W. Eason "Etch frustration in congruent lithium niobate single crystals induced by ferntosecond ultra-violet laser irradiation" Appl. Phys A (in press) 2001 